\title{
Revisiting Trilateration for Robot Localization
}

\author{
Federico Thomas and Lluís Ros
}

\begin{abstract}
Locating a robot from its distances, or range measurements, to three other known points or stations is a common operation, known as trilateration. This problem has been traditionally solved either by algebraic or numerical methods. An approach that avoids the direct algebrization of the problem is proposed here. Using constructive geometric arguments, a coordinate-free formula containing a small number of Cayley-Menger determinants is derived. This formulation accommodates a more thorough investigation of the effects caused by all possible sources of error, including round-off errors, for the first time in this context. New formulas for the variance and bias of the unknown robot location estimation, due to station location and range measurements errors, are derived and analyzed. They are proved to be more tractable compared with previous ones, because all their terms have geometric meaning, allowing a simple analysis of their asymptotic behavior near singularities.
\end{abstract}

Index Terms-Cayley-Menger determinants, error analysis, numerical conditioning, robot localization, trilateration.

\section{INTRODUCTION}

$\mathbf{T}$ RILATERATION is a method to determine the position of an object based on simultaneous range measurements from three stations located at known sites. This is a common operation not only in robot localization [20], but also in kinematics [2], [22], aeronautics [17], crystallography [16], and computer graphics [8]. It can be trivially expressed as the problem of finding the intersection of three spheres, that is, finding the solutions to the following system of quadratic equations:

$$
\left.\begin{array}{l}
\left(x-x_{1}\right)^{2}+\left(y-y_{1}\right)^{2}+\left(z-z_{1}\right)^{2}=l_{1}^{2} \\
\left(x-x_{2}\right)^{2}+\left(y-y_{2}\right)^{2}+\left(z-z_{2}\right)^{2}=l_{2}^{2} \\
\left(x-x_{3}\right)^{2}+\left(y-y_{3}\right)^{2}+\left(z-z_{3}\right)^{2}=l_{3}^{2}
\end{array}\right\}
$$

where $\mathbf{p}_{i}=\left(x_{i}, y_{i}, z_{i}\right), i=1,2,3$ are the coordinates of station $i$, and $l_{i}$ is the range measurement associated with it. In Fig. 1, thick segments between stations define the base plane, and thin ones, those connecting the moving object and the stations, correspond to the range measurements.

The problem of intersecting three spheres can be easily reduced to that of obtaining the intersection of a line and a sphere. Indeed, system (1) can be simplified into the following system of

Manuscript received September 26, 2003; revised May 4, 2004. This paper was recommended for publication by Associate Editor Z. Li and Editor S. Hutchinson upon evaluation of the reviewers' comments. This work was supported in part by the Spanish CICyT under Contract TIC2003-03396. The work of L. Ros was supported by a Ramón y Cajal contract from the Spanish Ministry of Education and Science.

The authors are with the Institut de Robòtica i Informàtica Industrial (CSICUPC), 08028 Barcelona, Spain (e-mail: fthomas@iri.upc.es; 1lros@iri.upc.es).

Digital Object Identifier 10.1109/TRO.2004.833793

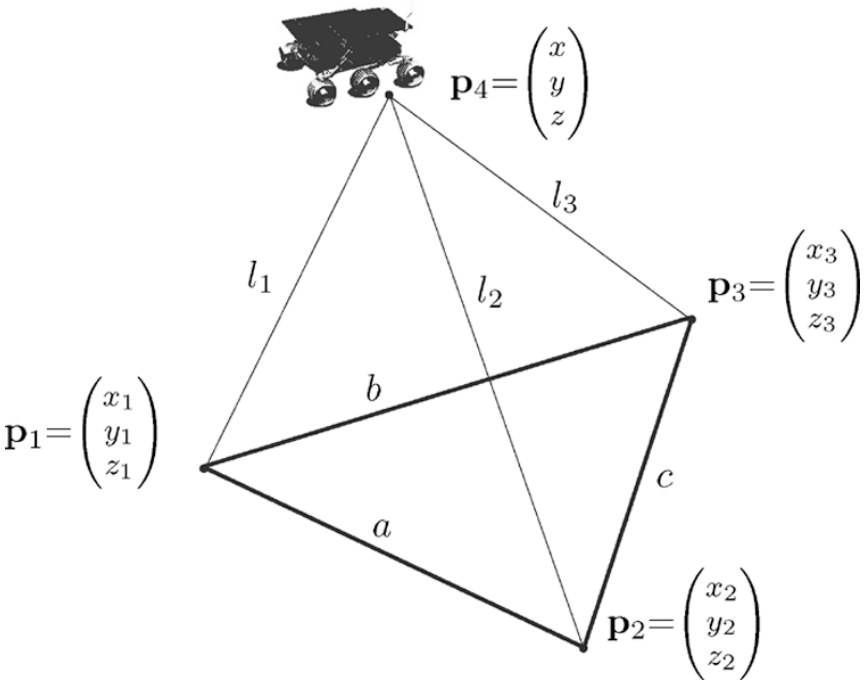

Fig. 1. The trilateration problem consists of obtaining the location of a mobile robot $\left(\mathbf{p}_{4}\right)$ from its distance to three stations (located at $\mathbf{p}_{1}, \mathbf{p}_{2}$, and $\mathbf{p}_{3}$ ).

two linear equations, whose solution is a line, and one quadratic equation:

$$
\left.\begin{array}{rl}
\left(x-x_{1}\right)^{2}+\left(y-y_{1}\right)^{2}+\left(z-z_{1}\right)^{2} & =l_{1}^{2} \\
x\left(x_{2}-x_{1}\right)+y\left(y_{2}-y_{1}\right)+z\left(z_{2}-z_{1}\right) & =\frac{\left(l_{1}^{2}-l_{2}^{2}+a^{2}\right)}{2} \\
x\left(x_{3}-x_{1}\right)+y\left(y_{3}-y_{1}\right)+z\left(z_{3}-z_{1}\right) & =\frac{\left(l_{1}^{2}-l_{3}^{2}+b^{2}\right)}{2}
\end{array}\right\}
$$

where $a=\left\|\mathbf{p}_{2}-\mathbf{p}_{1}\right\|$ and $b=\left\|\mathbf{p}_{3}-\mathbf{p}_{1}\right\|$.

The most straightforward way to obtain the two linear equations in (2) consists of subtracting the second and third equations from the first in (1), respectively, so that the quadratic terms cancel [8], [17], though other alternatives are possible [2].

Further simplifications are still possible by expressing the station coordinates according to a specific coordinate frame [9]. For example, by making the XY plane of the reference frame be the base plane, or making one coordinate axis coincide with the baseline between two stations, or simply locating the origin at one station. Nevertheless, this kind of simplification has an important drawback. If any other frame has to be used, a transformation has to be applied and, what is more important, the numerical conditioning of the resulting formulation depends on the chosen reference frame. This is why those formulations which are not linked to a particular reference frame, or coordinatefree formulations, are preferable. Available closed-form formulations of this kind directly take as input either system (1) [4] or (2) [17] and, despite their apparent simplicity, the expressions can be quite involved, in particular, those presented in [17], which are the standard formulas used in robotics [20]. In all cases, the results are obtained, apart from a square root, by employing standard techniques from linear algebra. 
As an alternative to closed-form formulations, there also exist numerical resolution methods for the trilateration problem. They take an approximation of the object position and iteratively achieve a better estimation by linearizing the measurement equations [10]. When analyzing what kind of influence the different sources of error have on the estimations, numerical approaches are of little help, so that closed-form formulations are obviously preferable.

In some applications, the range measurements or the station locations may not be known accurately, and this can lead to difficulties, particularly near singularities of the Jacobian of system (1), which correspond to locations in which the moving object is close to the base plane or the three stations are nearly aligned. Hereafter, these configurations will be referred to as singularities. In such cases, the problem can be formulated as a nonlinear least-squares problem to identify the best approximate solution [4]. Under these circumstances, it seems inevitable to rely on a numerical approach, because a closed-form formulation might yield no solution.

We propose here an alternative approach to previous closedform formulations that avoids the algebrization of the problem given by (1) or (2). Instead, by using barycentric coordinates, we derive a formula containing a few number of Cayley-Menger determinants, all of them having a geometric interpretation in terms of squared volumes, areas, or lengths. In this formulation, the station location coordinates appear explicitly as vectors, allowing a simple analysis of the effects caused by errors in these locations. In general, the analysis of how the different errors contribute to the estimation error, and how this error behaves near a singularity, is straightforward.

The paper is organized as follows. Section II presents some basic properties of Cayley-Menger determinants related to the geometry of tetrahedra, which are the key elements for the new vectorial coordinate-free solution to the trilateration problem presented in Section III. Based on this formulation, a complete error analysis of the trilateration operation is then given in Section IV. This analysis includes the study of the effects caused by range and station location errors in terms of variances and bias errors in the results. Section V is a digression on the minimization of the effects caused by roundoff errors when using limited computational resources and, finally, Section VI summarizes the main contributions and points that deserve further research.

\section{CAYley-Menger Determinants}

The Cayley-Menger bideterminant of two sequences of $n$ points, $\left[\mathbf{p}_{1}, \ldots, \mathbf{p}_{n}\right]$ and $\left[\mathbf{q}_{1}, \ldots, \mathbf{q}_{n}\right]$, is defined as

$$
\begin{aligned}
& D\left(\mathbf{p}_{1}, \ldots, \mathbf{p}_{n} ; \mathbf{q}_{1}, \ldots, \mathbf{q}_{n}\right) \\
& =2\left(\frac{-1}{2}\right)^{n}\left|\begin{array}{ccccc}
0 & 1 & 1 & 1 & 1 \\
1 & D\left(\mathbf{p}_{1}, \mathbf{q}_{1}\right) & D\left(\mathbf{p}_{1}, \mathbf{q}_{2}\right) & \cdots & D\left(\mathbf{p}_{1}, \mathbf{q}_{n}\right) \\
1 & D\left(\mathbf{p}_{2}, \mathbf{q}_{1}\right) & D\left(\mathbf{p}_{2}, \mathbf{q}_{2}\right) & \cdots & D\left(\mathbf{p}_{2}, \mathbf{q}_{n}\right) \\
\vdots & \vdots & \vdots & \ddots & \vdots \\
1 & D\left(\mathbf{p}_{n}, \mathbf{q}_{1}\right) & D\left(\mathbf{p}_{n}, \mathbf{q}_{2}\right) & \cdots & D\left(\mathbf{p}_{n}, \mathbf{q}_{n}\right)
\end{array}\right|
\end{aligned}
$$

where $D\left(\mathbf{p}_{i}, \mathbf{q}_{j}\right)$ denotes the squared distance between the points $\mathbf{p}_{i}$ and $\mathbf{q}_{j}$. This determinant plays a fundamental role in the so-called "distance geometry," a term coined by Blumenthal in [1] which refers to the analytical study of Euclidean geometry in terms of invariants, without resorting to artificial coordinate systems. Since in many cases of interest the two sequences of points are the same, it will be convenient to abbreviate $D\left(\mathbf{p}_{1}, \ldots, \mathbf{p}_{n} ; \mathbf{p}_{1}, \ldots, \mathbf{p}_{n}\right)$ by $D\left(\mathbf{p}_{1}, \ldots, \mathbf{p}_{n}\right)$, which is simply called a Cayley-Menger determinant. Next, we give the geometric interpretation of these determinants for $n=2,3,4$. For further details, the reader is referred to [6, pp. 126-129] and [12].

As for the Cayley-Menger determinants, it can be shown that $D\left(\mathbf{p}_{1}, \ldots, \mathbf{p}_{n}\right)$ is $((n-1) !)^{2}$ times the squared hypervolume of the simplex spanned by the points $\mathbf{p}_{1}, \ldots, \mathbf{p}_{n}$ in $\Re^{n-1}$. Hence, for $n=2$

$$
D\left(\mathbf{p}_{1}, \mathbf{p}_{2}\right)=d\left(\mathbf{p}_{1}, \mathbf{p}_{2}\right)^{2}
$$

where $d\left(\mathbf{p}_{1}, \mathbf{p}_{2}\right)$ is the Euclidean distance between $\mathbf{p}_{1}$ and $\mathbf{p}_{2}$. Observe that the use of the symbol $D\left(\mathbf{p}_{i}, \mathbf{p}_{j}\right)$ for both the squared distance from $\mathbf{p}_{1}$ to $\mathbf{p}_{2}$ and their Cayley-Menger determinant is thus consistent.

For $n=3$, if $A$ is the area of the triangle spanned by $\mathbf{p}_{1}, \mathbf{p}_{2}$, and $\mathbf{p}_{3}$, we obtain Herron's formula relating $A$ with the side lengths

$$
D\left(\mathbf{p}_{1}, \mathbf{p}_{2}, \mathbf{p}_{3}\right)=4 A^{2}=\left\|\left(\mathbf{p}_{2}-\mathbf{p}_{1}\right) \times\left(\mathbf{p}_{3}-\mathbf{p}_{1}\right)\right\|^{2} .
$$

For $n=4$, if $V$ is the volume of the tetrahedron spanned by $\mathbf{p}_{1}, \mathbf{p}_{2}, \mathbf{p}_{3}$, and $\mathbf{p}_{4}$, we obtain Euler's formula, relating $V$ to the edge lengths

$$
D\left(\mathbf{p}_{1}, \mathbf{p}_{2}, \mathbf{p}_{3}, \mathbf{p}_{4}\right)=36 V^{2} .
$$

For the Cayley-Menger bideterminants, it can be shown that, for $n=2$

$$
D\left(\mathbf{p}_{1}, \mathbf{p}_{2} ; \mathbf{q}_{1}, \mathbf{q}_{2}\right)=\left(\mathbf{p}_{1}-\mathbf{p}_{2}\right) \cdot\left(\mathbf{q}_{1}-\mathbf{q}_{2}\right) .
$$

Since this dot product can be expressed as $d\left(\mathbf{p}_{1}, \mathbf{p}_{2}\right) d\left(\mathbf{q}_{1}, \mathbf{q}_{2}\right) \cos (\theta)$, with $\theta$ being the angle between the lines supporting the segments $\mathbf{p}_{1} \mathbf{p}_{2}$ and $\mathbf{q}_{1} \mathbf{q}_{2}$, this yields the following formula for $\cos (\theta)$, in terms of the six interpoint distances:

$$
\cos \theta=\frac{D\left(\mathbf{p}_{1}, \mathbf{p}_{2} ; \mathbf{q}_{1}, \mathbf{q}_{2}\right)}{D\left(\mathbf{p}_{1}, \mathbf{p}_{2}\right) D\left(\mathbf{q}_{1}, \mathbf{q}_{2}\right)} .
$$

By expanding $D\left(\mathbf{p}_{1}, \mathbf{p}_{2} ; \mathbf{q}_{1}, \mathbf{q}_{2}\right)$ in terms of the involved distances, the reader can easily see that when $\mathbf{p}_{1}=\mathbf{q}_{1}$, this formula reduces to the law of cosines for a triangle.

Likewise, for $n=3$, it can be shown that

$$
\begin{aligned}
& D\left(\mathbf{p}_{1}, \mathbf{p}_{2}, \mathbf{p}_{3} ; \mathbf{q}_{1}, \mathbf{q}_{2}, \mathbf{q}_{3}\right) \\
& \quad=\left(\left(\mathbf{p}_{1}-\mathbf{p}_{3}\right) \times\left(\mathbf{p}_{2}-\mathbf{p}_{3}\right)\right) \cdot\left(\left(\mathbf{q}_{1}-\mathbf{q}_{3}\right) \times\left(\mathbf{q}_{2}-\mathbf{q}_{3}\right)\right) .
\end{aligned}
$$

The right-hand side (RHS) of this equation can be easily shown to be equal to $4 A_{1} A_{2} \cdot \cos (\phi)$, where $A_{1}$ and $A_{2}$ are the areas of the triangles $\mathbf{p}_{1} \mathbf{p}_{2} \mathbf{p}_{3}$ and $\mathbf{q}_{1} \mathbf{q}_{2} \mathbf{q}_{3}$, respectively, 


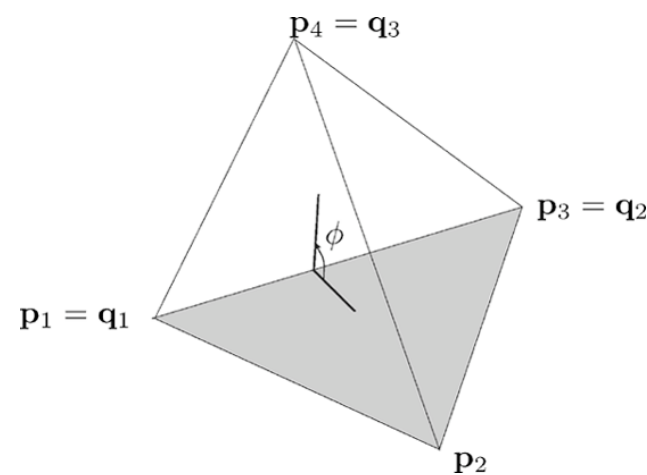

Fig. 2. An interior dihedral angle of the tetrahedron defined by $\mathbf{p}_{1}, \mathbf{p}_{2}, \mathbf{p}_{3}$, and $\mathbf{p}_{4}$.

and $\phi$ is the dihedral angle between the planes they define. By expressing these areas as Cayley-Menger determinants of the triangles' vertices, this yields the following formula for the cosine of $\phi$, in terms of interpoint distances:

$$
\cos \phi=\frac{D\left(\mathbf{p}_{1}, \mathbf{p}_{2}, \mathbf{p}_{3} ; \mathbf{q}_{1}, \mathbf{q}_{2}, \mathbf{q}_{3}\right)}{D^{\frac{1}{2}}\left(\mathbf{p}_{1}, \mathbf{p}_{2}, \mathbf{p}_{3}\right) D^{\frac{1}{2}}\left(\mathbf{q}_{1}, \mathbf{q}_{2}, \mathbf{q}_{3}\right)}
$$

which can be regarded as the law of cosines generalized to a tetrahedron when $\mathbf{q}_{1}=\mathbf{p}_{1}$ and $\mathbf{q}_{2}=\mathbf{p}_{3}$ (see Fig. 2). In other words

$$
\cos ^{2} \phi=\frac{D^{2}\left(\mathbf{p}_{1}, \mathbf{p}_{2}, \mathbf{p}_{3} ; \mathbf{p}_{1}, \mathbf{p}_{3}, \mathbf{p}_{4}\right)}{D\left(\mathbf{p}_{1}, \mathbf{p}_{2}, \mathbf{p}_{3}\right) D\left(\mathbf{p}_{1}, \mathbf{p}_{3}, \mathbf{p}_{4}\right)} .
$$

An alternative formulation for the law of cosines generalized to a tetrahedron can be found in [15], which permits alternatively expressing $\cos ^{2} \phi$ as

$$
\cos ^{2} \phi=1-\frac{D\left(\mathbf{p}_{1}, \mathbf{p}_{2}, \mathbf{p}_{3}, \mathbf{p}_{4}\right) D\left(\mathbf{p}_{1}, \mathbf{p}_{3}\right)}{D\left(\mathbf{p}_{1}, \mathbf{p}_{2}, \mathbf{p}_{3}\right) D\left(\mathbf{p}_{1}, \mathbf{p}_{3}, \mathbf{p}_{4}\right)} .
$$

Identifying the RHSs of (6) and (7), we get

$$
\begin{array}{r}
D^{2}\left(\mathbf{p}_{1}, \mathbf{p}_{2}, \mathbf{p}_{3} ; \mathbf{p}_{1}, \mathbf{p}_{3}, \mathbf{p}_{4}\right)=D\left(\mathbf{p}_{1}, \mathbf{p}_{2}, \mathbf{p}_{3}\right) D\left(\mathbf{p}_{1}, \mathbf{p}_{3}, \mathbf{p}_{4}\right) \\
-D\left(\mathbf{p}_{1}, \mathbf{p}_{2}, \mathbf{p}_{3}, \mathbf{p}_{4}\right) D\left(\mathbf{p}_{1}, \mathbf{p}_{3}\right)
\end{array}
$$

which will be useful later.

Finally, for $n=4$, the bideterminant is equal to the product of two triple products

$$
\begin{aligned}
& D\left(\mathbf{p}_{1}, \mathbf{p}_{2}, \mathbf{p}_{3}, \mathbf{p}_{4} ; \mathbf{q}_{1}, \mathbf{q}_{2}, \mathbf{q}_{3}, \mathbf{q}_{4}\right) \\
& \quad=\left|\mathbf{p}_{1}-\mathbf{p}_{4}, \mathbf{p}_{2}-\mathbf{p}_{4}, \mathbf{p}_{3}-\mathbf{p}_{4}\right| \cdot\left|\mathbf{q}_{1}-\mathbf{q}_{4}, \mathbf{q}_{2}-\mathbf{q}_{4}, \mathbf{q}_{3}-\mathbf{q}_{4}\right|
\end{aligned}
$$

and hence, it can be interpreted as 36 times the product of the volumes of the tetrahedra $\mathbf{p}_{1}, \mathbf{p}_{2}, \mathbf{p}_{3}, \mathbf{p}_{4}$, and $\mathbf{q}_{1}, \mathbf{q}_{2}, \mathbf{q}_{3}, \mathbf{q}_{4}$.

\section{A NEW FORMULATION FOR TRILATERATION}

Given three points in space, say $\mathbf{p}_{1}, \mathbf{p}_{2}$, and $\mathbf{p}_{3}$, the trilateration problem consists of finding the location of another point, say $\mathbf{p}_{4}$, whose distance to these three points is known. According to Fig. 3, using barycentric coordinates [5, pp. 216-221], the location of the orthogonal projection of $\mathbf{p}_{4}$ onto the base, say $\mathbf{p}$, can be expressed as

$$
\mathbf{p}=\frac{A_{1} \mathbf{p}_{1}+A_{2} \mathbf{p}_{2}+A_{3} \mathbf{p}_{3}}{A_{b}}
$$

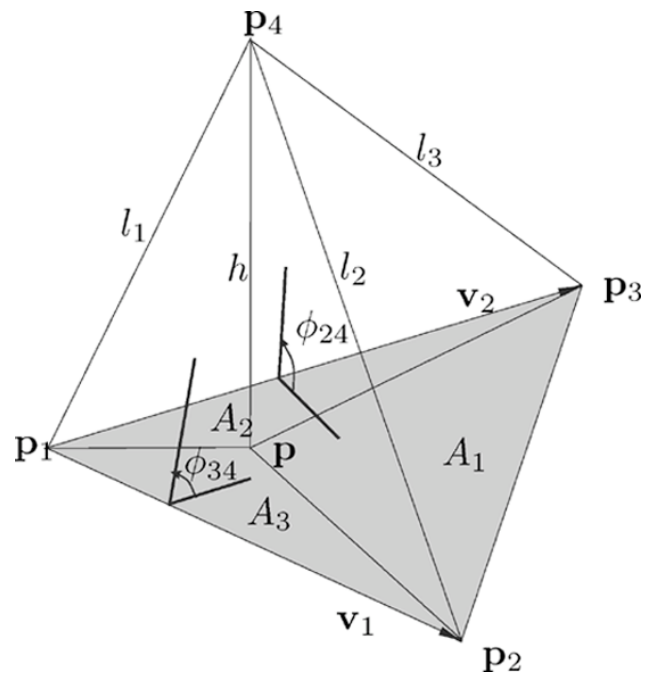

Fig. 3. Barycentric coordinates of the projection of $\mathbf{p}_{4}$ onto the plane defined by $\mathbf{p}_{1}, \mathbf{p}_{2}$, and $\mathbf{p}_{3}$.

where $A_{1}, A_{2}$, and $A_{3}$ are the signed areas ${ }^{1}$ of the triangles $\mathbf{p}_{2} \mathbf{p}_{3} \mathbf{p}, \mathbf{p}_{3} \mathbf{p}_{1} \mathbf{p}$, and $\mathbf{p}_{1} \mathbf{p}_{2} \mathbf{p}$, respectively, and $A_{b}$ is the area of the triangle $\mathbf{p}_{1} \mathbf{p}_{2} \mathbf{p}_{3}$. Alternatively

$$
\mathbf{p}=\mathbf{p}_{1}+\frac{A_{2}}{A_{b}} \mathbf{v}_{1}+\frac{A_{3}}{A_{b}} \mathbf{v}_{2}
$$

where $\mathbf{v}_{1}=\mathbf{p}_{2}-\mathbf{p}_{1}$ and $\mathbf{v}_{2}=\mathbf{p}_{3}-\mathbf{p}_{1}$.

The values $A_{i}$ can be obtained by projecting the areas of the triangles coincident in $\mathbf{p}_{4}$, onto the base plane. Hence, using (3)

$$
\begin{aligned}
& \frac{A_{2}}{A_{b}}=+\sqrt{\frac{D\left(\mathbf{p}_{1}, \mathbf{p}_{3}, \mathbf{p}_{4}\right)}{D\left(\mathbf{p}_{1}, \mathbf{p}_{2}, \mathbf{p}_{3}\right)}} \cos \left(\phi_{24}\right) \\
& \frac{A_{3}}{A_{b}}=+\sqrt{\frac{D\left(\mathbf{p}_{1}, \mathbf{p}_{2}, \mathbf{p}_{4}\right)}{D\left(\mathbf{p}_{1}, \mathbf{p}_{2}, \mathbf{p}_{3}\right)}} \cos \left(\phi_{34}\right)
\end{aligned}
$$

where $\phi_{24}$ and $\phi_{34}$ are the dihedral angles indicated in Fig. 3. Moreover, using (5), we can write

$$
\mathbf{p}=\mathbf{p}_{1}+k_{1} \mathbf{v}_{1}+k_{2} \mathbf{v}_{2}
$$

where

$$
\begin{aligned}
& k_{1}=-\frac{D\left(\mathbf{p}_{1}, \mathbf{p}_{2}, \mathbf{p}_{3} ; \mathbf{p}_{1}, \mathbf{p}_{3}, \mathbf{p}_{4}\right)}{D\left(\mathbf{p}_{1}, \mathbf{p}_{2}, \mathbf{p}_{3}\right)} \\
& k_{2}=\frac{D\left(\mathbf{p}_{1}, \mathbf{p}_{2}, \mathbf{p}_{3} ; \mathbf{p}_{1}, \mathbf{p}_{2}, \mathbf{p}_{4}\right)}{D\left(\mathbf{p}_{1}, \mathbf{p}_{2}, \mathbf{p}_{3}\right)}
\end{aligned}
$$

Now, $\mathbf{p}_{4}$ can be obtained as

$$
\mathbf{p}_{4}=\mathbf{p} \pm k_{3}\left(\mathbf{v}_{1} \times \mathbf{v}_{2}\right)
$$

where the \pm sign accounts for the two mirror symmetric locations of $\mathbf{p}_{4}$ with respect to the base plane, and $k_{3}$ is equal to the height $h$ of the tetrahedron, divided by the norm of $\mathbf{v}_{1} \times \mathbf{v}_{2}$.

${ }^{1}$ For a triangle pqr in the Euclidean plane with area $A$, the signed area is defined as $+A$ (respectively, $-A$ ) if the point $\mathbf{q}$ is to the right (resp. to the left) of the line $\mathbf{p r}$, when going from $\mathbf{p}$ to $\mathbf{r}$. 
Since the volume of the tetrahedron is $(1 / 3) A_{b} h$, using (3) and (4), we can write

$$
h=+\sqrt{\frac{D\left(\mathbf{p}_{1}, \mathbf{p}_{2}, \mathbf{p}_{3}, \mathbf{p}_{4}\right)}{D\left(\mathbf{p}_{1}, \mathbf{p}_{2}, \mathbf{p}_{3}\right)}} .
$$

Moreover, again using (3)

$$
\left\|\mathbf{v}_{1} \times \mathbf{v}_{2}\right\|=\sqrt{D\left(\mathbf{p}_{1}, \mathbf{p}_{2}, \mathbf{p}_{3}\right)}
$$

one concludes that

$$
k_{3}=\frac{+\sqrt{D\left(\mathbf{p}_{1}, \mathbf{p}_{2}, \mathbf{p}_{3}, \mathbf{p}_{4}\right)}}{D\left(\mathbf{p}_{1}, \mathbf{p}_{2}, \mathbf{p}_{3}\right)} .
$$

Hence, the final expression for $\mathbf{p}_{4}$ is

$$
\begin{aligned}
\mathbf{p}_{4}= & \mathbf{p}_{1}+\frac{1}{D\left(\mathbf{p}_{1}, \mathbf{p}_{2}, \mathbf{p}_{3}\right)} \\
& \cdot\left(-D\left(\mathbf{p}_{1}, \mathbf{p}_{2}, \mathbf{p}_{3} ; \mathbf{p}_{1}, \mathbf{p}_{3}, \mathbf{p}_{4}\right) \cdot \mathbf{v}_{1}\right. \\
& +D\left(\mathbf{p}_{1}, \mathbf{p}_{2}, \mathbf{p}_{3} ; \mathbf{p}_{1}, \mathbf{p}_{2}, \mathbf{p}_{4}\right) \cdot \mathbf{v}_{2} \\
& \left. \pm \sqrt{D\left(\mathbf{p}_{1}, \mathbf{p}_{2}, \mathbf{p}_{3}, \mathbf{p}_{4}\right)} \cdot\left(\mathbf{v}_{1} \times \mathbf{v}_{2}\right)\right)
\end{aligned}
$$

This formula can be easily rewritten in matrix form as

$\mathbf{p}_{4}=\mathbf{A} \cdot\left(l_{1}, l_{2}, l_{3}\right)^{t} \pm \mathbf{c} \sqrt{\left(l_{1}, l_{2}, l_{3}, l_{1}^{2}, l_{2}^{2}, l_{3}^{2}, l_{1} l_{2}, l_{1} l_{3}, l_{2} l_{3}\right) \mathbf{b}}$

where $\mathbf{A}, \mathbf{b}$, and $\mathbf{c}$ are appropriate constant matrices and vectors involving cofactors of $D\left(\mathbf{p}_{1}, \mathbf{p}_{2}, \mathbf{p}_{3} ; \mathbf{p}_{1}, \mathbf{p}_{3}, \mathbf{p}_{4}\right)$, $D\left(\mathbf{p}_{1}, \mathbf{p}_{2}, \mathbf{p}_{3} ; \mathbf{p}_{1}, \mathbf{p}_{2}, \mathbf{p}_{4}\right)$, and $D\left(\mathbf{p}_{1}, \mathbf{p}_{2}, \mathbf{p}_{3}, \mathbf{p}_{4}\right)$. This form coincides with Manolakis' expression, given in [17], considered as the computationally most efficient formula for trilateration and used in robot localization [20]. While obtaining (13) from (12) is straightforward, the converse is by no means obvious.

The main advantage of (12) over (13) is that it is mathematically more tractable, because all terms are determinants with geometric meaning. For example, (12) permits realizing that only when $D\left(\mathbf{p}_{1}, \mathbf{p}_{2}, \mathbf{p}_{3}\right)=0$, i.e., when $\mathbf{p}_{1}, \mathbf{p}_{2}$, and $\mathbf{p}_{3}$ are aligned, the location of $\mathbf{p}_{4}$ is undefined, and only when $D\left(\mathbf{p}_{1}, \mathbf{p}_{2}, \mathbf{p}_{3}, \mathbf{p}_{4}\right)=0$, i.e., when $\mathbf{p}_{4}$ lies on the base plane, the solution for $\mathbf{p}_{4}$ is unique. This kind of reasoning cannot be carried out on (13).

\section{ERROR ANALYSIS}

This section shows how (12) accommodates a more thorough error analysis than previously done for the trilateration problem. Under the assumption that both the station locations and range measurements are corrupted by zero-mean uncorrelated random noise with a Gaussian probability density function, explicit expressions for the variance and bias errors of the object location estimation are obtained in terms of squared distances, areas, and volumes.

\section{A. Station Location Errors}

The station location error analysis given in [18] requires the inversion of the Jacobian matrix resulting from the linearization of system (1). Unfortunately, this inversion becomes ill-conditioned near a singularity. We can take full advantage of (12), though, to avoid this inversion. Since (12) is linear with respect to each station location, it allows us to prove that when station locations errors are the only source of error, the object location estimation is unbiased, despite all involved nonlinearities. Also, its associated covariance matrix has a simple expression in terms of lengths, areas, and volumes.

Let $\delta \mathbf{p}_{i}$ and $\mathbf{p}_{i}^{0}$ denote the additive random error and the actual value of station location $\mathbf{p}_{i}, i=1,2,3$, respectively. Then, $\mathbf{p}_{i}=\mathbf{p}_{i}^{0}+\delta \mathbf{p}_{i}$. These errors are assumed to have zero-mean value, that is $E\left\{\delta \mathbf{p}_{i}\right\}=0$, where $E\{\cdot\}$ stands for the expected value operation. We also assume that their three coordinates are uncorrelated with the same variance $\sigma_{p}^{2}$ for the three stations. In other words

$$
E\left\{\delta \mathbf{p}_{i} \delta \mathbf{p}_{j}^{t}\right\}= \begin{cases}\sigma_{p}^{2} \mathbf{I}, & \text { if } i=j \\ E\left\{\delta \mathbf{p}_{i}\right\} E\left\{\delta \mathbf{p}_{j}^{t}\right\}=\mathbf{0}, & \text { if } i \neq j\end{cases}
$$

where I denotes the identity matrix. Then, using (12), it is easy to check that

$$
\begin{aligned}
\delta \mathbf{p}_{4}= & \mathbf{p}_{4}-\mathbf{p}_{4}^{0} \\
= & \delta \mathbf{p}_{1}+k_{1} \delta \mathbf{v}_{1}+k_{2} \delta \mathbf{v}_{2} \\
& \pm k_{3}\left[\left(\mathbf{v}_{1}^{0} \times \delta \mathbf{v}_{2}\right)+\left(\delta \mathbf{v}_{1} \times \mathbf{v}_{2}^{0}\right)+\left(\delta \mathbf{v}_{1} \times \delta \mathbf{v}_{2}\right)\right]
\end{aligned}
$$

where $\delta \mathbf{v}_{1}=\delta \mathbf{p}_{2}-\delta \mathbf{p}_{1}$ and $\delta \mathbf{v}_{2}=\delta \mathbf{p}_{3}-\delta \mathbf{p}_{1}$. Then, the bias error due to the error in the location of the stations can be stated as follows:

$$
\begin{aligned}
E\left\{\delta \mathbf{p}_{4}\right\} & = \pm k_{3} E\left\{\delta \mathbf{v}_{1} \times \delta \mathbf{v}_{2}\right\} \\
& = \pm k_{3} E\left\{\left(\delta \mathbf{p}_{1} \times \delta \mathbf{p}_{2}\right)+\left(\delta \mathbf{p}_{2} \times \delta \mathbf{p}_{3}\right)+\left(\delta \mathbf{p}_{3} \times \delta \mathbf{p}_{1}\right)\right\}
\end{aligned}
$$

which is identically zero because all scalar products required to compute the above cross products involve uncorrelated random variables (reminding that if two Gaussian variables, say $a$ and $b$, are uncorrelated, they are independent, that is, $E(a b)=E(a) E(b))$.

In turn, the covariance matrix of the position estimate error $\delta \mathbf{p}_{4}$ can be evaluated as

$$
\begin{aligned}
C_{s} & =E\left\{\left[\delta \mathbf{p}_{4}-E\left\{\delta \mathbf{p}_{4}\right\}\right]\left[\delta \mathbf{p}_{4}-E\left\{\delta \mathbf{p}_{4}\right\}\right]^{t}\right\} \\
& =E\left\{\delta \mathbf{p}_{4} \delta \mathbf{p}_{4}^{t}\right\}-E\left\{\delta \mathbf{p}_{4}\right\} E\left\{\delta \mathbf{p}_{4}^{t}\right\} \\
& =E\left\{\delta \mathbf{p}_{4} \delta \mathbf{p}_{4}^{t}\right\} .
\end{aligned}
$$

Then, after expanding the above expected value operation and removing expected values involving products of uncorrelated variables, we have

$$
\begin{aligned}
C_{s}= & \left(1-k_{1}-k_{2}\right)^{2} E\left\{\delta \mathbf{p}_{1} \delta \mathbf{p}_{1}^{t}\right\} \\
& +k_{1}^{2} E\left\{\delta \mathbf{p}_{2} \delta \mathbf{p}_{2}^{t}\right\}+k_{2}^{2} E\left\{\delta \mathbf{p}_{3} \delta \mathbf{p}_{3}^{t}\right\} \\
& +k_{3}^{2} E\left\{\left(\mathbf{v}_{1}^{0} \times \delta \mathbf{p}_{3}\right)\left(\mathbf{v}_{1}^{0} \times \delta \mathbf{p}_{3}\right)^{t}\right\} \\
& +k_{3}^{2} E\left\{\left(\mathbf{v}_{2}^{0} \times \delta \mathbf{p}_{2}\right)\left(\mathbf{v}_{2}^{0} \times \delta \mathbf{p}_{2}\right)^{t}\right\} \\
& +k_{3}^{2} E\left\{\left(\mathbf{v}_{3}^{0} \times \delta \mathbf{p}_{1}\right)\left(\mathbf{v}_{3}^{0} \times \delta \mathbf{p}_{1}\right)^{t}\right\}
\end{aligned}
$$

where $\mathbf{v}_{3}^{0}=\mathbf{p}_{3}^{0}-\mathbf{p}_{1}^{0}$. That is

$$
C_{s}=\sigma_{p}^{2}\left(k_{1}^{2}+k_{2}^{2}+\left(1-k_{1}-k_{2}\right)^{2}+2 k_{3}^{2}\left(a^{2}+b^{2}+c^{2}\right)\right) \mathbf{I}
$$

where $a=\left\|\mathbf{p}_{2}-\mathbf{p}_{1}\right\|, b=\left\|\mathbf{p}_{3}-\mathbf{p}_{1}\right\|$, and $c=\left\|\mathbf{p}_{4}-\mathbf{p}_{1}\right\|$. 


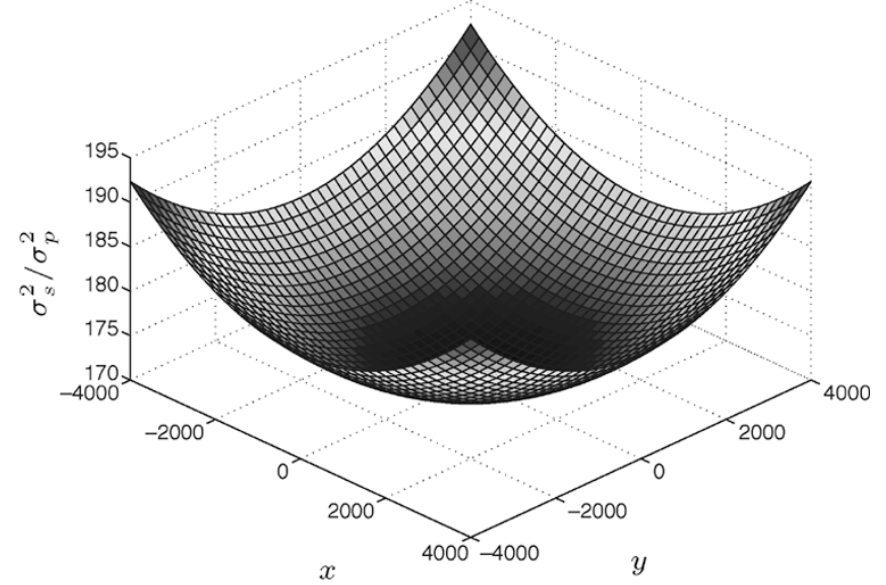

(a)

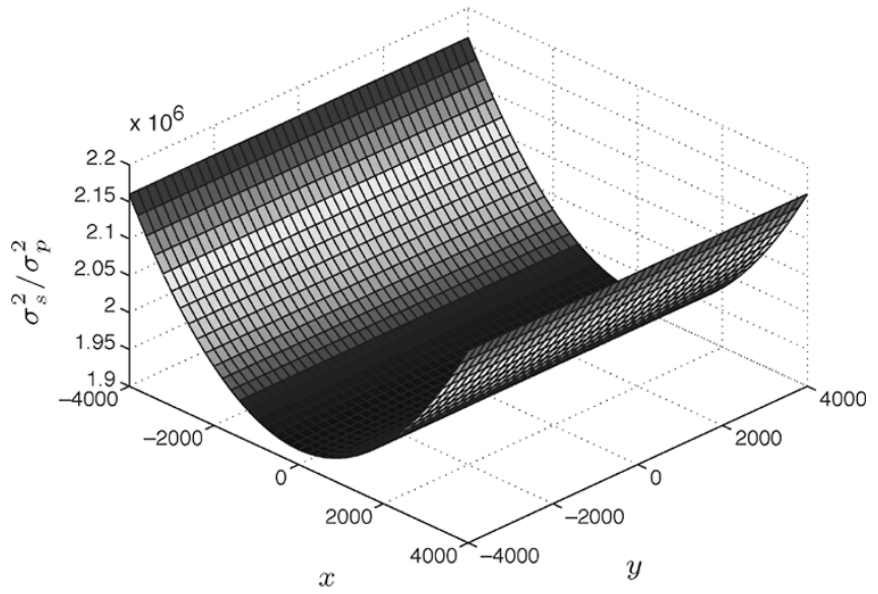

(b)

Fig. 4. Relative error $\left(\sigma_{s}^{2} / \sigma_{p}^{2}\right)$ in the robot location for two representative cases (see text for details).

The resulting covariance matrix is isotropic: the variance in the robot location is the same along any direction, say $\sigma_{s}^{2}$. Then

$$
\sigma_{s}^{2}=\sigma_{p}^{2}\left(k_{1}^{2}+k_{2}^{2}+\left(1-k_{1}-k_{2}\right)^{2}+2 k_{3}^{2}\left(a^{2}+b^{2}+c^{2}\right)\right) \text {. }
$$

Finally, in terms of volume, areas, and lengths, we have

$$
\left(\frac{\sigma_{s}}{\sigma_{p}}\right)^{2}=\frac{1}{A_{b}^{2}}\left(A_{1}^{2}+A_{2}^{2}+A_{3}^{2}\right)+\frac{18 V^{2}}{A_{b}^{4}}\left(a^{2}+b^{2}+c^{2}\right) \text {. }
$$

Assuming that the three stations are not aligned, for high distances to the base plane, (18) can be approximated by

$$
\left.\left(\frac{\sigma_{s}}{\sigma_{p}}\right)^{2}\right|_{V \rightarrow \infty} \simeq \frac{18 V^{2}}{A_{b}^{4}}\left(a^{2}+b^{2}+c^{2}\right)
$$

and for low distances by

$$
\left.\left(\frac{\sigma_{s}}{\sigma_{p}}\right)^{2}\right|_{V \rightarrow 0} \simeq \frac{1}{A_{b}^{2}}\left(A_{1}^{2}+A_{2}^{2}+A_{3}^{2}\right) .
$$

Fig. 4 examines this relative error for two representative cases. In the first case, the three stations form an equilateral triangle on the $\mathrm{XY}$ plane inscribed in a circle centered in the origin of radius 1000 distance units. The locations of the stations are $\mathbf{p}_{1}=(-500 \sqrt{3},-500,0)^{t}, \mathbf{p}_{2}=(0,1000,0)^{t}$, and $\mathbf{p}_{3}=(500 \sqrt{3},-500,0)^{t}$. The data-acquisition area of the system is a square area at a distance of 8000 units from the base plane, spanning in each direction from -4000 to 4000 units [see Fig. 4(a)]. In the second case, the stations are located at $\mathbf{p}_{1}=(-500,-5,0)^{t}, \mathbf{p}_{2}=(0,5,0)^{t}$, and $\mathbf{p}_{3}=(500,-5,0)^{t}$, i.e., they are almost aligned along the $\mathrm{X}$ axis. The acquisition area in this case is on the base plane itself [see Fig. 4(b)]. We observe that while in Fig. 4(a), the error increases as we move away from the stations' barycenter, in Fig. 4(b), a privileged direction of low error arises coinciding with the axis along which the sum $A_{1}^{2}+A_{2}^{2}+A_{3}^{2}$ in (20) is minimum.

Although the error analysis given in [18] is not carried out for Gaussian error distributions, the provided results are consistent with the results obtained here for the first case. The results of the second case cannot be compared with previous results, because it corresponds to a singularity in which the robot is located on the base plane, which cannot be treated by previous formulations.

\section{B. Range Measurement Errors}

The error analysis given here for the range measurements is parallel to that presented in [17]. We adapt it to our formulation, showing that the same results can be obtained in a more concise form.

Let $\delta l_{i}$ and $l_{i}^{0}$ denote the additive random error and the actual value of range measurement $l_{i}$, respectively. Let $\delta \mathbf{l}=\left(\delta l_{1}, \delta l_{2}, \delta l_{3}\right)^{t}$ and $\mathbf{l}^{0}=\left(l_{1}^{0}, l_{2}^{0}, l_{3}^{0}\right)^{t}$. Then, $\mathbf{l}=\mathbf{l}^{0}+\delta \mathbf{l}$.

The range errors are assumed to have zero-mean value, that is $E\{\delta \mathbf{1}\}=0$. We also assume that they are uncorrelated with the same variance $\sigma_{r}^{2}$. Consequently, their covariance matrix can be expressed as

$$
E\left\{\delta \mathbf{l} \delta \mathbf{l}^{t}\right\}=\sigma_{r}^{2} \mathbf{I} .
$$

For small range errors, the robot location can be well approximated by retaining the terms up to the second-order partial derivatives in the Taylor expansion of (9), that is

$$
\delta \mathbf{p}_{4}=\mathbf{p}_{4}-\mathbf{p}_{4}^{0}=\sum_{i=1}^{3} \frac{\partial \mathbf{p}_{4}}{\partial l_{i}} \delta l_{i}+\frac{1}{2} \sum_{i=1}^{3} \sum_{j=1}^{3} \frac{\partial^{2} \mathbf{p}_{4}}{\partial l_{i} \partial l_{j}} \delta l_{i} \delta l_{j} .
$$

Then the expected value of robot location error, i.e., the bias error, is

$$
E\left\{\delta \mathbf{p}_{4}\right\}=\frac{1}{2} \sum_{i=1}^{3} \sum_{j=1}^{3} \frac{\partial^{2} \mathbf{b}_{1}}{\partial l_{i} \partial l_{j}} E\left\{\delta l_{i} \delta l_{j}\right\} .
$$

Using (21)

$$
E\left\{\delta \mathbf{p}_{4}\right\}=\frac{\sigma_{r}^{2}}{2}\left(\frac{\partial^{2} \mathbf{p}_{4}}{\partial l_{1}^{2}}+\frac{\partial^{2} \mathbf{p}_{4}}{\partial l_{2}^{2}}+\frac{\partial^{2} \mathbf{p}_{4}}{\partial l_{3}^{2}}\right) .
$$

Finally, substituting (12)

$$
E\left\{\delta \mathbf{p}_{4}\right\}=\frac{\sigma_{r}^{2}}{2}\left(\nabla^{2} k_{1} \mathbf{v}_{1}+\nabla^{2} k_{2} \mathbf{v}_{2} \pm \nabla^{2} k_{3}\left(\mathbf{v}_{1} \times \mathbf{v}_{2}\right)\right)
$$

where $\nabla^{2} k_{i}=\left(\partial^{2} k_{i} / \partial l_{1}^{2}\right)+\left(\partial^{2} k_{i} / \partial l_{2}^{2}\right)+\left(\partial^{2} k_{i} / \partial l_{3}^{2}\right)$, and the + or - sign is used, depending on the chosen trilateration 
solution. The analytic expression for these derivatives can be found in Appendix I.

As we already mentioned, the trilateration bias error was already examined in [17], where two main results were drawn:

(R1) projection of the bias error onto the base plane can be neglected;

(R2) bias error becomes relevant as the robot location approaches the base plane.

Contrary to all other formulations, these two facts have a direct accommodation in ours. It is important to realize that $\nabla^{2} k_{1}$ and $\nabla^{2} k_{2}$ are constant, i.e., the bias error parallel to the base plane is constant, independent of the robot location. This contradicts, in part, the results presented in [17]. Nevertheless, this has no practical effects because, as a consequence of (R1), $\nabla^{2} k_{1}$ and $\nabla^{2} k_{2}$ can be neglected in front of $\nabla^{2} k_{3}$, and (22) can be approximated by

$$
E\left\{\delta \mathbf{p}_{4}\right\} \simeq \pm \frac{\sigma_{r}^{2}}{2} \nabla^{2} k_{3}\left(\mathbf{v}_{1} \times \mathbf{v}_{2}\right) .
$$

As a consequence of (R2), this can be further simplified, for low distances to the base plane, into (see Appendix I for the details)

$$
\left.E\left\{\delta \mathbf{p}_{4}\right\}\right|_{V \rightarrow 0} \simeq \mp \sigma_{r}^{2} \frac{l_{1}^{2} A_{3}^{2}+l_{2}^{2} A_{2}^{2}+l_{3}^{2} A_{1}^{2}}{54 V^{3}}\left(\mathbf{v}_{1} \times \mathbf{v}_{2}\right) .
$$

This equation is remarkable because of its simplicity, when compared with its counterpart in [17].

It can be checked that, as a consequence of this error, when the robot moves on a plane parallel to the base plane, the estimation will erroneously indicate that it increases and decreases its distance to this plane when it approaches, and goes away from, the barycenter of the stations, respectively.

Fig. 5 examines this error for the first representative case used in the previous section. The maximum bias error in the considered acquisition area is $-0.03 \sigma_{r}^{2}$ (Fig. 5, top). This amount exponentially increases as the robot reduces its distance to the base plane, i.e., the singularity is approached. When the distance is 400 , the maximum bias error is $-12 \sigma_{r}^{2}$, and when it is reduced to 40 , the maximum bias error mounts to $-11842 \sigma_{r}^{2}$ (Fig. 5, middle). These results fully agree with those presented in [17].

\section{A Digression ON NUMERICAL ERRORS}

Although (12) and (13) are algebraically equivalent, their behavior in the presence of roundoff of errors could be quite different. Actually, near a singularity, one can even obtain different results with the same formulation just by scaling data or permuting indexes. Nevertheless, in practice, fine results can be obtained using any formulation by evaluating their subexpressions in double precision if all involved values are given in single precision [13].

This section shows how the effect of round-off errors can be minimized during the evaluation of (12) by using Kahan's factorizations. This has interest when the involved evaluations cannot be performed in higher precision than that of the data because of limited computational resources.

The analysis of the numerical stability of (12) can be reduced to that of evaluating Cayley-Menger determinants. Although no
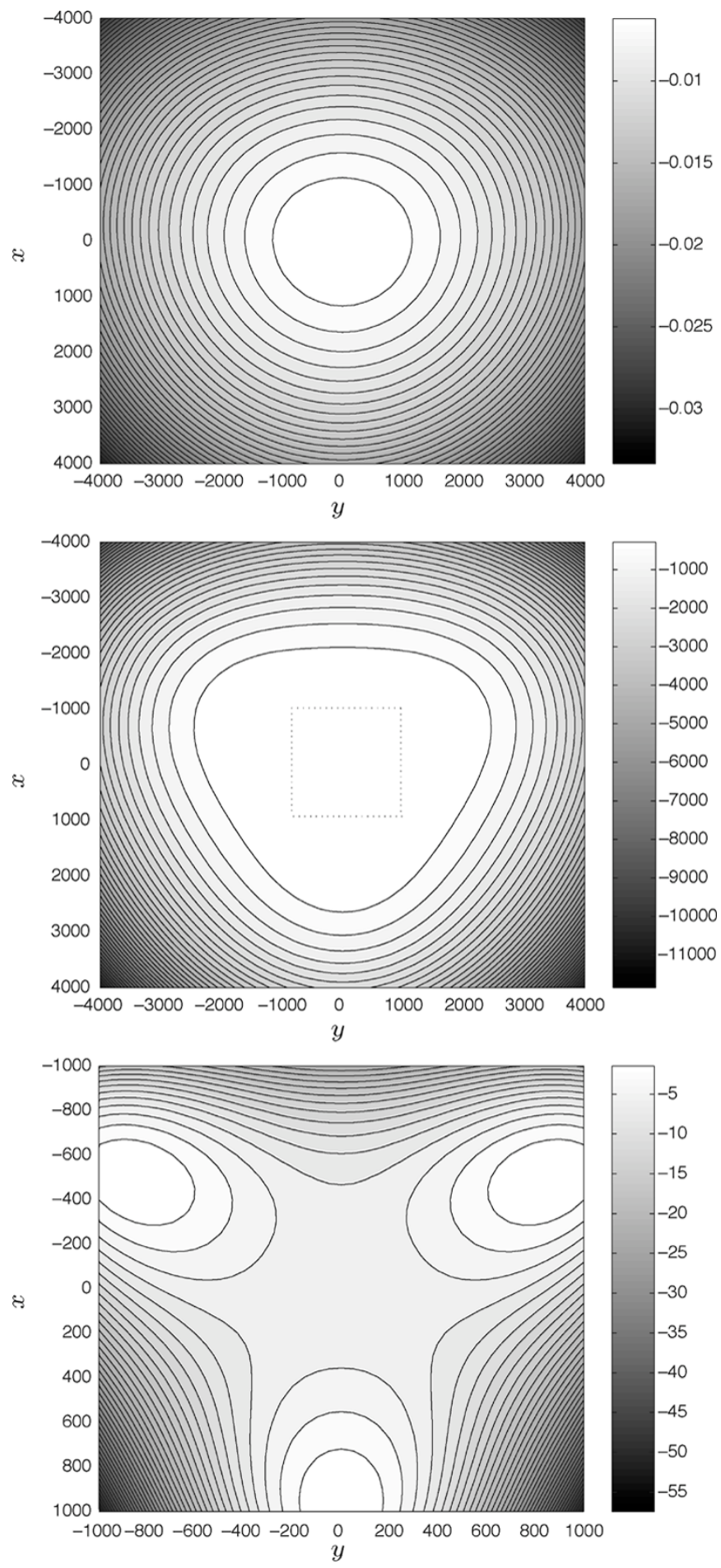

Fig. 5. Component of the trilateration bias error orthogonal to the base plane, normalized with respect to $\sigma_{r}^{2}$, for a representative example where the distance to the base plane is 4000 (top) and 40 (middle) distance units. The bottom plot shows an enlargement of the dotted region shown in the middle plot.

general numerically stable method for evaluating determinants is known to the authors, nor satisfactory error bounds for their evaluation having been proposed [11, p. 13], extensive research has been done in the stable evaluation of Cayley-Menger determinants of the form $D(\mathbf{a}, \mathbf{b}, \mathbf{c})$ and $D(\mathbf{a}, \mathbf{b}, \mathbf{c}, \mathbf{d})$ [13]. Before reviewing the results presented therein, it is worth giving a general idea about the nature and complexity of the problem. 
The direct expansion of a $n \times n$ determinant leads to $n$ ! terms. The way these terms are added is fundamental to attaining high numerical stability. Following the discussion given in [11], consider the substraction, in exact arithmetic, $\hat{x}=\hat{a}-\hat{b}$, where $\hat{a}=a(1+\triangle a)$ and $\hat{b}=b(1+\triangle b)$. The terms $\triangle a$ and $\triangle b$ are relative errors or uncertainties in the data, perhaps attributable to previous computations. With $x=a-b$, we have

$$
\left|\frac{x-\hat{x}}{x}\right|=\left|\frac{-a \triangle a+b \triangle b}{a-b}\right| \leq \max (|\triangle a|,|\triangle b|) \frac{|a|+|b|}{|a-b|} .
$$

Hence, the relative error for $\hat{x}$ is large when $|a-b| \ll|a|+|b|$, that is, when there is a heavy cancellation in the substraction. This shows that subtractive cancellation causes relative errors or uncertainties already present in $\hat{a}$ and $\hat{b}$ to be magnified. Then, to reduce the effect of cancellation, a summation like $(x+y-z)$ can be computed from an expression like

$$
(\max \{x, y\}-z)+\min \{x, y\} .
$$

At this point, one comes up with two possible solutions for evaluating a Cayley-Menger determinant: 1) designing a summation method aiming at minimizing the effects of cancellation of each intermediate sum [11, p. 82], or 2) factorizing the determinant in as many factors as possible and computing each of them so that cancellation is minimized. The latter is not only preferable because the former is NP-hard [14], but also because Cayley-Menger determinants of the form $D(\mathbf{a}, \mathbf{b}, \mathbf{c})$ and $D(\mathbf{a}, \mathbf{b}, \mathbf{c}, \mathbf{d})$ can be decomposed into factors that can be evaluated using (25). Appendix II compiles these factorizations due to Kahan [13].

To complete a trilateration operation, it remains to accurately compute Cayley-Menger bideterminants of the form $\Xi=D(\mathbf{a}, \mathbf{b}, \mathbf{c} ; \mathbf{b}, \mathbf{c}, \mathbf{d})$. Using (8), it turns out that

$$
\Xi=\operatorname{sign}(\Xi) \sqrt{D(\mathbf{a}, \mathbf{b}, \mathbf{c}) D(\mathbf{b}, \mathbf{c}, \mathbf{d})-D(\mathbf{a}, \mathbf{b}, \mathbf{c}, \mathbf{d}) D(\mathbf{b}, \mathbf{c})} .
$$

Then, since the square-root part can be computed using Kahan's factorizations, the problem is reduced to that of accurately computing the sign of a determinant. Fortunately, the question of how to be sure that the sign is determined correctly in floatingpoint arithmetics has surfaced and been solved in the field of computational geometry (see [21] and the references therein).

\section{CONCLUSIONS}

An alternative closed-form formulation for trilateration based on constructive geometric arguments, not algebraic, has been presented. The result is a formula containing a few Cayley-Menger determinants. It is more general than that presented in [17], which is considered as the computationally most efficient, because it can be easily derived from ours.

It has been shown how all algebraic manipulations based on our formulation can be performed involving only distances, and the results can always be interpreted in terms of distances, areas, and volumes. This has been revealed of great interest when calculating the partial derivatives of the robot location with respect to the range measurements, allowing remarkable simple formulas for the covariances and bias errors due to station location and range errors, respectively. Their asymptotic behavior near a singularity, due either to the alignment of the three stations or the proximity of the robot to the base plane, has been shown to be easily derivable.

It has also been shown how, using our formulation and Kahan's factorizations, the effects caused by roundoff errors can be minimized.

The presented error analysis has been carried out for static measurements, but, in general, trilateration is used for estimating trajectories; that is, for tracking purposes. The measurements along a trajectory are not statistically uncorrelated, so they should be jointly smoothed during tracking to improve accuracy using, for example, a Kalman filter. The error analysis given in this paper is of relevance to this end. For example, the characterization of the bias error must not be ignored at this point, and it has to be suitably anticipated in this filter. Also, our error analysis could be of interest for optimal estimation of the solution when more than three stations are involved. These issues deserve further research.

\section{APPENDIX I}

This appendix is devoted to computing second derivatives of $k_{1}, k_{2}$, and $k_{3}$ with respect to $l_{1}, l_{2}$, and $l_{3}$. To start with, note that since the denominator in

$$
k_{1}=-\frac{D\left(\mathbf{p}_{1}, \mathbf{p}_{2}, \mathbf{p}_{3} ; \mathbf{p}_{1}, \mathbf{p}_{3}, \mathbf{p}_{4}\right)}{D\left(\mathbf{p}_{1}, \mathbf{p}_{2}, \mathbf{p}_{3}\right)}
$$

does not depend on $l_{1}, l_{2}$, or $l_{3}$, then

$$
\frac{\partial^{2} k_{1}}{\partial l_{j}^{2}}=\frac{-1}{D\left(\mathbf{p}_{1}, \mathbf{p}_{2}, \mathbf{p}_{3}\right)} \cdot \frac{\partial^{2} D\left(\mathbf{p}_{1}, \mathbf{p}_{2}, \mathbf{p}_{3} ; \mathbf{p}_{1}, \mathbf{p}_{3}, \mathbf{p}_{4}\right)}{\partial l_{j}^{2}}
$$

and we only need the second derivatives of

$$
\begin{aligned}
& D\left(\mathbf{p}_{1}, \mathbf{p}_{2}, \mathbf{p}_{3} ; \mathbf{p}_{1}, \mathbf{p}_{3}, \mathbf{p}_{4}\right) \\
& =-\frac{1}{4}\left|\begin{array}{cccc}
0 & 1 & 1 & 1 \\
1 & 0 & D\left(\mathbf{p}_{1}, \mathbf{p}_{3}\right) & l_{1}^{2} \\
1 & D\left(\mathbf{p}_{1}, \mathbf{p}_{2}\right) & D\left(\mathbf{p}_{2}, \mathbf{p}_{3}\right) & l_{2}^{2} \\
1 & D\left(\mathbf{p}_{1}, \mathbf{p}_{3}\right) & 0 & l_{3}^{2}
\end{array}\right|
\end{aligned}
$$

with respect to $l_{1}, l_{2}$, and $l_{3}$. By expanding this determinant by the last column, we easily realize that

$$
\begin{aligned}
& \frac{\partial D\left(\mathbf{p}_{1}, \mathbf{p}_{2}, \mathbf{p}_{3} ; \mathbf{p}_{1}, \mathbf{p}_{3}, \mathbf{p}_{4}\right)}{\partial l_{1}}=-D\left(\mathbf{p}_{2}, \mathbf{p}_{3} ; \mathbf{p}_{1}, \mathbf{p}_{3}\right) l_{1} \\
& \frac{\partial D\left(\mathbf{p}_{1}, \mathbf{p}_{2}, \mathbf{p}_{3} ; \mathbf{p}_{1}, \mathbf{p}_{3}, \mathbf{p}_{4}\right)}{\partial l_{2}}=D\left(\mathbf{p}_{1}, \mathbf{p}_{3}\right) l_{2} \\
& \frac{\partial D\left(\mathbf{p}_{1}, \mathbf{p}_{2}, \mathbf{p}_{3} ; \mathbf{p}_{1}, \mathbf{p}_{3}, \mathbf{p}_{4}\right)}{\partial l_{3}}=-D\left(\mathbf{p}_{1}, \mathbf{p}_{2} ; \mathbf{p}_{1}, \mathbf{p}_{3}\right) l_{3}
\end{aligned}
$$

and, hence, that

$$
\begin{aligned}
\frac{\partial^{2} k_{1}}{\partial l_{1}^{2}} & =\frac{D\left(\mathbf{p}_{2}, \mathbf{p}_{3} ; \mathbf{p}_{1}, \mathbf{p}_{3}\right)}{D\left(\mathbf{p}_{1}, \mathbf{p}_{2}, \mathbf{p}_{3}\right)} \\
\frac{\partial^{2} k_{1}}{\partial l_{2}^{2}} & =-\frac{D\left(\mathbf{p}_{1}, \mathbf{p}_{3}\right)}{D\left(\mathbf{p}_{1}, \mathbf{p}_{2}, \mathbf{p}_{3}\right)} \\
\frac{\partial^{2} k_{1}}{\partial l_{3}^{2}} & =\frac{D\left(\mathbf{p}_{1}, \mathbf{p}_{2} ; \mathbf{p}_{1}, \mathbf{p}_{3}\right)}{D\left(\mathbf{p}_{1}, \mathbf{p}_{2}, \mathbf{p}_{3}\right)} .
\end{aligned}
$$


Then, after substituting the above determinants by their geometric interpretations

$$
\nabla^{2} k_{1}=\frac{b^{2}}{8 A_{b}^{2}}
$$

Proceeding in an analogous way for $k_{2}$, we obtain

$$
\begin{aligned}
\frac{\partial^{2} k_{2}}{\partial l_{1}^{2}} & =-\frac{D\left(\mathbf{p}_{2}, \mathbf{p}_{3} ; \mathbf{p}_{1}, \mathbf{p}_{2}\right)}{D\left(\mathbf{p}_{1}, \mathbf{p}_{2}, \mathbf{p}_{3}\right)} \\
\frac{\partial^{2} k_{2}}{\partial l_{2}^{2}} & =\frac{D\left(\mathbf{p}_{1}, \mathbf{p}_{3} ; \mathbf{p}_{1}, \mathbf{p}_{2}\right)}{D\left(\mathbf{p}_{1}, \mathbf{p}_{2}, \mathbf{p}_{3}\right)} \\
\frac{\partial^{2} k_{2}}{\partial l_{3}^{2}} & =-\frac{D\left(\mathbf{p}_{1}, \mathbf{p}_{2}\right)}{D\left(\mathbf{p}_{1}, \mathbf{p}_{2}, \mathbf{p}_{3}\right)} .
\end{aligned}
$$

Then

$$
\nabla^{2} k_{2}=\frac{a^{2}}{8 A_{b}^{2}}
$$

With regard to the second derivatives of $k_{3}$, it can easily be checked that by applying the chain rule to

$$
k_{3}=\frac{\sqrt{D\left(\mathbf{p}_{1}, \mathbf{p}_{2}, \mathbf{p}_{3}, \mathbf{p}_{4}\right)}}{D\left(\mathbf{p}_{1}, \mathbf{p}_{2}, \mathbf{p}_{3}\right)}
$$

we get

$$
\frac{\partial^{2} k_{3}}{\partial l_{j}^{2}}=\frac{1}{48 A_{b}^{2} V} \eta-\frac{1}{3456 A_{b}^{2} V^{3}} \mu^{2}
$$

where $\eta \quad=\quad\left(\partial^{2} D\left(\mathbf{p}_{1}, \mathbf{p}_{2}, \mathbf{p}_{3}, \mathbf{p}_{4}\right) / \partial l_{j}^{2}\right)$ and $\mu=\left(\partial D\left(\mathbf{p}_{1}, \mathbf{p}_{2}, \mathbf{p}_{3}, \mathbf{p}_{4}\right) / \partial l_{j}\right)$. Then, it only remains to compute the first and second derivatives of $D\left(\mathbf{p}_{1}, \mathbf{p}_{2}, \mathbf{p}_{3}, \mathbf{p}_{4}\right)$ with respect to the desired length. To this end, we write

$D\left(\mathbf{p}_{1}, \mathbf{p}_{2}, \mathbf{p}_{3}, \mathbf{p}_{4}\right)$

$$
=\frac{1}{8}\left|\begin{array}{ccccc}
0 & 1 & 1 & 1 & 1 \\
1 & 0 & D\left(\mathbf{p}_{1}, \mathbf{p}_{2}\right) & D\left(\mathbf{p}_{1}, \mathbf{p}_{3}\right) & l_{1}^{2} \\
1 & D\left(\mathbf{p}_{1}, \mathbf{p}_{2}\right) & 0 & D\left(\mathbf{p}_{2}, \mathbf{p}_{3}\right) & l_{2}^{2} \\
1 & D\left(\mathbf{p}_{1}, \mathbf{p}_{3}\right) & D\left(\mathbf{p}_{2}, \mathbf{p}_{3}\right) & 0 & l_{3}^{2} \\
1 & l_{1}^{2} & l_{2}^{2} & l_{3}^{2} & 0
\end{array}\right|
$$

and realize that

$$
\begin{aligned}
& \frac{\partial D\left(\mathbf{p}_{1}, \mathbf{p}_{2}, \mathbf{p}_{3}, \mathbf{p}_{4}\right)}{\partial l_{1}}=2 l_{1} D\left(\mathbf{p}_{1}, \mathbf{p}_{2}, \mathbf{p}_{3} ; \mathbf{p}_{3}, \mathbf{p}_{2}, \mathbf{p}_{4}\right) \\
& \frac{\partial D\left(\mathbf{p}_{1}, \mathbf{p}_{2}, \mathbf{p}_{3}, \mathbf{p}_{4}\right)}{\partial l_{2}}=2 l_{2} D\left(\mathbf{p}_{1}, \mathbf{p}_{2}, \mathbf{p}_{3} ; \mathbf{p}_{1}, \mathbf{p}_{3}, \mathbf{p}_{4}\right) \\
& \frac{\partial D\left(\mathbf{p}_{1}, \mathbf{p}_{2}, \mathbf{p}_{3}, \mathbf{p}_{4}\right)}{\partial l_{3}}=2 l_{3} D\left(\mathbf{p}_{1}, \mathbf{p}_{2}, \mathbf{p}_{3} ; \mathbf{p}_{2}, \mathbf{p}_{1}, \mathbf{p}_{4}\right) .
\end{aligned}
$$

Then, proceeding as for $k_{1}$ and $k_{2}$, we get

$$
\begin{aligned}
& \frac{\partial D^{2}\left(\mathbf{p}_{1}, \mathbf{p}_{2}, \mathbf{p}_{3}, \mathbf{p}_{4}\right)}{\partial l_{1}^{2}} \\
& =2 D\left(\mathbf{p}_{1}, \mathbf{p}_{2}, \mathbf{p}_{3} ; \mathbf{p}_{3}, \mathbf{p}_{2}, \mathbf{p}_{4}\right)+2 l_{1}^{2} c^{2} \\
& \frac{\partial D^{2}\left(\mathbf{p}_{1}, \mathbf{p}_{2}, \mathbf{p}_{3}, \mathbf{p}_{4}\right)}{\partial l_{2}^{2}} \\
& =2 D\left(\mathbf{p}_{1}, \mathbf{p}_{2}, \mathbf{p}_{3} ; \mathbf{p}_{1}, \mathbf{p}_{3}, \mathbf{p}_{4}\right)+2 l_{2}^{2} b^{2} \\
& \frac{\partial D^{2}\left(\mathbf{p}_{1}, \mathbf{p}_{2}, \mathbf{p}_{3}, \mathbf{p}_{4}\right)}{\partial l_{3}^{2}} \\
& =2 D\left(\mathbf{p}_{1}, \mathbf{p}_{2}, \mathbf{p}_{3} ; \mathbf{p}_{2}, \mathbf{p}_{1}, \mathbf{p}_{4}\right)+2 l_{3}^{2} a^{2}
\end{aligned}
$$

Finally, after substituting (29) and (30) into (28), and expressing the result in terms of volumes, areas, and lengths, we conclude that

$$
\nabla^{2} k_{3}=\frac{1}{6 V}+\frac{l_{1}^{2} c^{2}+l_{2}^{2} b^{2}+l_{3}^{2} c^{2}}{24 A_{b}^{2} V}-\frac{l_{1}^{2} A_{3}^{2}+l_{2}^{2} A_{2}^{2}+l_{3}^{2} A_{1}^{2}}{54 V^{3}} .
$$

If the three stations are nearly aligned, then $A_{b} \rightarrow 0$ and

$$
\left.\nabla^{2} k_{3}\right|_{A_{b} \rightarrow 0} \simeq \frac{l_{1}^{2} c^{2}+l_{2}^{2} b^{2}+l_{3}^{2} c^{2}}{24 A_{b}^{2} V} .
$$

If the three stations are not aligned but the object's location is near the base plane, then $V \rightarrow 0$ and

$$
\left.\nabla^{2} k_{3}\right|_{V \rightarrow 0} \simeq-\frac{l_{1}^{2} A_{3}^{2}+l_{2}^{2} A_{2}^{2}+l_{3}^{2} A_{1}^{2}}{54 V^{3}} .
$$

\section{APPENDIX II}

This appendix summarizes Kahan's factorizations for $D\left(\mathbf{p}_{1}, \mathbf{p}_{2}, \mathbf{p}_{3}\right)$ and $D\left(\mathbf{p}_{1}, \mathbf{p}_{2}, \mathbf{p}_{3}, \mathbf{p}_{4}\right)$ [13].

According to the notation used in Fig. 1, we have that

$$
D\left(\mathbf{p}_{1}, \mathbf{p}_{2}, \mathbf{p}_{3}\right)=(a+b+c)(b-c+a)(c-a+b)(a-b+c)
$$

and

$$
\begin{aligned}
D\left(\mathbf{p}_{1}, \mathbf{p}_{2}, \mathbf{p}_{3}, \mathbf{p}_{4}\right)= & \frac{1}{1024 l_{1}^{2} l_{2}^{2} l_{3}^{2}}(\xi+\eta+\zeta-\lambda)(\lambda+\xi+\eta-\zeta) \\
& \times(\eta+\zeta+\lambda-\xi)(\zeta+\lambda+\xi-\eta)
\end{aligned}
$$

where

$$
\xi=\sqrt{x Y Z}, \quad \eta=\sqrt{y Z X}, \quad \zeta=\sqrt{z X Y}, \quad \lambda=\sqrt{x y z}
$$

and

$$
\begin{aligned}
X & =\left(l_{1}-a+l_{2}\right)\left(a+l_{2}+l_{1}\right) \\
Y & =\left(l_{3}-b+l_{1}\right)\left(b+l_{1}+l_{3}\right) \\
Z & =\left(l_{2}-c+l_{3}\right)\left(c+l_{3}+l_{2}\right) \\
x & =\left(a-l_{2}+l_{1}\right)\left(l_{2}-l_{1}+a\right) \\
y & =\left(b-l_{1}+l_{3}\right)\left(l_{1}-l_{3}+b\right) \\
z & =\left(c-l_{3}+l_{2}\right)\left(l_{3}-l_{2}+c\right) .
\end{aligned}
$$

By permuting data, other factorizations are possible. The nine factors of the form $(x-y+z)$ in (36) are called facial differences. The total number of facial differences is 12 , but the above factorization only uses nine. The factorization is numerically stable, provided that the smallest of the 12 facial differences lie among the nine used.

\section{REFERENCES}

[1] L. M. Blumenthal, Theory and Applications of Distance Geometry. Oxford, U.K.: Oxford Univ. Press, 1953.

[2] H. Bruyninckx, "Forward kinematics for Hunt-Primrose parallel manipulators," Mech. Mach. Theory, vol. 34, pp. 657-664, 1999.

[3] A. Cayley, "A theorem in the geometry of position," Cambridge Math. J., vol. II, pp. 267-271, 1841.

[4] I. D. Coope, "Reliable computation of the points of intersection of $n$ spheres in $\Re^{n}$," Australian, New Zealand Ind. Appl. Math. J., pt. C, vol. 42, pp. 461-477, 2000.

[5] H. S. M. Coxeter, Introduction to Geometry. New York: Wiley, 1989.

[6] G. M. Crippen and T. Havel, Distance Geometry and Molecular Conformation. New York: Wiley, 1988. 
[7] H. Dörrie, 100 Great Problems of Elementary Mathematics. Their History and Solution. New York: Dover, 1965.

[8] D. Eberly, Finding the intersection of three spheres, comp.graphics.algorithms newsgroup, 1996.

[9] B. T. Fang, "Trilateration and extension to global positioning system navigation," J. Guidance, Contr., Dynam., vol. 9, no. 6, pp. 715-717, 1986.

[10] W. H. Foy, "Position-location solutions by Taylor-series estimation," IEEE Trans. Aerosp. Electron. Syst., vol. AES-12, pp. 187-194, Feb. 1976.

[11] N. J. Higham, Accuracy and Stability of Numerical Algorithms, 2nd ed. Philadelphia: SIAM, 2002.

[12] T. Havel, "Some examples of the use of distances as coordinates in Euclidean geometry," J. Symbolic Computat., no. 11, pp. 579-593, 1991.

[13] W. Kahan. What has the volume of a tetrahedron to do with computer programming languages?. Univ. California, Berkeley, CA. [Online]. Available: http://www.cs.berkeley.edu/ whahan/VtetLang.pdf

[14] M.-Y. Kao and J. Wang, "Linear-time approximation algorithms for computing numerical summation with probably small error," SIAM J. Comput., vol. 29, no. 5, pp. 1568-1576, 2000

[15] J. R. Lee, "The law of cosines in a tetrahedron," J. Korea Soc. Math Ed.: Pure Appl. Math., ser. B, no. 4, pp. 1-6, 1997.

[16] A. L. Mackay, "Generalized structural geometry," Acta Crystalographica, vol. A-30, pp. 440-447, 1974.

[17] D. E. Manolakis, "Efficient solution and performance analysis of 3-D position estimation by trilateration," IEEE Trans. Aerosp. Electron. Syst., vol. 32, pp. 1239-1248, Apr. 1996

[18] D. E. Manolakis and M. E. Cox, "Effect in range difference position estimation due to stations' position errors," IEEE Trans. Aerosp. Electron. Syst., vol. 34, pp. 329-334, Jan. 1998.

[19] K. Menger, "New foundation for Euclidean geometry," Amer. J. Math., no. 53, pp. 721-745, 1931.

[20] L. E. Navarro-Serment, C. J. J. Paredis, and P. Khosla, "A beacon system for the localization of distributed robotic teams," in Proc. Int. Conf. Field and Service Robots, Pittsburgh, PA, Aug. 29-31, 1999, pp. 232-237.

[21] V. Y. Pan and Y. Yu, "Certified computation of the sign of a matrix determinant," Algorithmica, vol. 30, no. 4, pp. 715-724, 2001.
[22] F. Thomas, E. Ottaviano, L. Ros, and M. Ceccarelli, "Coordinate-free formulation of a 3-2-1 wire-base tracking system using Cayley-Menger determinants," in Proc. IEEE Int. Conf. Robotics and Automation, vol. 1, Taipei, Taiwan, 2003, pp. 255-363.

[23] A. Cayley, "A theorem in the geometry of position," in Collected Mathematical Papers of Arthur Cayley. Cambridge, U.K.: Cambridge Univ. Press, 1963.

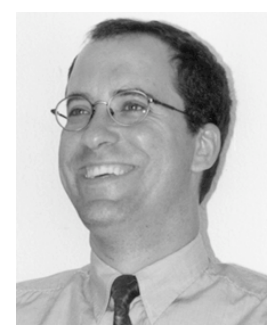

Federico Thomas received the B.Sc. degree in telecommunications engineering in 1984 and the $\mathrm{Ph} . \mathrm{D}$. degree (with honors) in computer science in 1988, both from the Technical University of Catalonia, Barcelona, Spain.

Since March 1990, he has been a Research Scientist with the Industrial Robotics Institute of the Spanish High Council for Scientific Research, Barcelona, Spain. His research interests are in geometry and kinematics, with applications to robotics, computer graphics and machine vision.

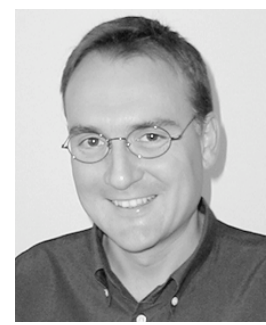

Lluís Ros received the mechanical engineering degree in 1992, and the Ph.D. degree (with honors) in industrial engineering in 2000, both from the Technical University of Catalonia, Barcelona, Spain.

From 1993 to 1996, he worked with the Control of Resources Group of the Cybernetics Institute, Barcelona, involved in the application of constraint logic programming to the control of electric and water networks. Since August 2000, he has been a Research Scientist with the Industrial Robotics Institute of the Spanish High Council for Scientific Research. His current research interests are in geometry and kinematics, with applications to robotics, computer graphics and machine vision. 